\title{
Computational Modeling of Peptide - Aptamer Binding in Biosensor Applications
}

\author{
Kristen L. Rhinehardt, Ram V. Mohan, Goundla Srinivas, and Ajit D. Kelkar
}

\begin{abstract}
Biomarkers are molecules that indicate changes in a physiological state and are detected by biosensors. Aptamer based biosensors are highly efficient, with high specificity and reusability. The present paper focuses on Molecular Dynamics (MD) modeling to simulate, analyze, and subsequently visualize the aptamer binding combination of mucin 1(MUC1) peptide, a breast cancer biomarker, and Anti-MUC1 aptamer. Analysis indicated that the peptide associated twice with this aptamer. In particular, the peptide associated with the $12^{\text {th }}$ tyrosine residue of the aptamer loop after 25ns before dissociating and binding with the 3' and 5' ends of the aptamer. Post simulation analysis of the radius of gyration, atomic distance to the wet lab surface plasma resonance imaging (SPRi) results corroborated with the observations of the simulation results. Computational molecular dynamics simulations can provide molecular level insight for aptamer-peptide binding process, which is difficult to probe directly in wet lab experiments.
\end{abstract}

Index Terms-Biosensors, biomarkers, peptide-aptamer binding, molecular dynamics modeling.

\section{INTRODUCTION}

Biomarkers are molecules that correspond with bio-chemical changes in the body. Biomarkers undergo changes in concentration, physiology and morphology as they track disease progression and drug effectiveness in the body. A biosensor is a receptor-transducer device that provides quantitative information using a bio-recognition element and a transducer. The transducer is based on electrochemical, mass, optical or thermal principles while the bio-recognition element or bio-receptor acts on some biochemical mechanism. The working of the bio-recognition element of the biosensor is based on the formation of the biological complex. When a biological sample is loaded into the sensor, the bio-recognition element/bio-receptor recognizes the target or "key" in the sample and binds to it. The transducer registers the change which is quantified and displayed for the user. Difficulty in sensor development occurs with picking a proper bio-recognition element.

The most common method of making biosensors is using antibodies as the bio-recognition elements. Though antibodies are large molecules that are specific in their binding, they are not readily synthesized and can be chemically unstable. In addition, their size can create low

Manuscript received May 27, 2013; revised July 27, 2013. This work was supported in part by the U.S. Army Research Office under Grant W991NF1110168.

Kristen L. Rhinehardt, Ram V. Mohan, Goundla Srinivas, and Ajit D. Kelkar are with Department of Nanoengineering, Joint School of Nanoscience and Nanoengineering, North Carolina A\&T State University, Greensboro, NC 27401, USA (e-mail: rvmohan@ncat.edu). density, single use biosensors that can cause errors and inaccuracies in readings. Another possible type of bio-recognition elements are aptamers. Aptamers are oligonucleotides sequences made of single stranded DNA or RNA. Aptamers are advantageous as a bio-receptor since they are small, chemically stable and have a high binding affinity that rival or better than that of antibodies. This high binding affinity is not only due to their ability to bind to a structure but also to fold in correspondence to that binding. Aptamers can also be easily functionalized and immobilized to surfaces to create highly ordered receptor layers [1].

A compilation of these oligonucleotides has been made into aptamer libraries. These aptamers can be made from DNA or RNA and stored in an aptamer library. A standard 25-mer library compilation currently stands at $10^{15}$ available aptamers. The size of an aptamer library depends on the length of the variable region and can be approximated as

$$
\text { library size }=4^{n}
$$

where $n$ is the length of the variable region in the aptamer (1). In 1990, a reasonable experimental solution has been provided by the Systematic Evolution of Ligands by Exponential Enrichment (SELEX) process, in which developed libraries undergo incubation with the desired target molecule [2]. Those that bind are enhanced through PCR (polymerase chain reaction) and the process repeats until a small group of high affinity aptamers remain. These aptamers are then sequenced to de-cover their makeup.

Over the years, the potential for the applicability of breast cancer biomarkers into a biosensor have garnished a lot of interest. Significant research investigations at the genetic and molecular level for this type of cancer have been conducted [3]. One gene in particular that is unregulated in the breast cancer cells is the MUC1 gene [3]. This gene codes for the trans-membrane protein MUC1. Trans-membrane mucin proteins are thought to be sensors for environmental changes and as a second line of defense [4] lack the tandem repeat regions [3], [4] and has O-glycans which are shorter and expose the core of the protein that contains a variety of peptide epitopes. In breast cancer there is an up regulation of the MUC1/Y isoform that lacks the tandem repeat region and is free floating in the blood [3]. The antibody, SM3, that has a high affinity for this version of MUC1 and has been crystallized with a peptide antigen APDTRPAP that is exposed in the carcinogenic isoform of MUC1 creating a protein-peptide complex [5]. There is an inverse relationship between antibody concentration and disease severity. Those with a decreased severity of breast cancer will have more free antibodies than bound antibodies. Higher concentrations of 
free antibody have been shown to inhibit distant metastases. Such concentrations could be quantified within a biosensor. Selecting an aptamer and determining how best to implant it in a biosensor can be time consuming and unclear using wet lab techniques. Current research has shown that MUC1 peptide and anti-MUC1 aptamer do indeed bind. However, the orientation of binding between the unbound MUC1 peptide and aptamer is unknown. This paper focuses on computational modeling of Mucin 1 (MUC 1) peptide and Anit-MUC1 aptamer binding for their fundamental understanding. Computational modeling of this protein aptamer binding provides a methodology for such analysis that is of relevance to associated biosensor applications.

\section{Computational Modeling}

Computational modeling that improves our understanding of the potential target molecules could greatly facilitate the selection of target molecules for any biomarker; could aid in making an efficient diagnostic tool for a given disease. Computational modeling could thus facilitate the identification of the potential high probability target molecule candidates for the focused biomarkers and understand their behavior and bindings for the enhancement of biosensor devices. Of particular interest is in the understanding of the molecular level binding between the peptide and aptamer systems through molecular level modeling of the associated molecular constituents. The power of today's computational modeling provides the ability to test, analyze and visualize the aptamer binding that forms the basis of the aptamer selection process for biosensor applications, and provides the foundation and methodology in the present work.

The multitude of large library of potential aptamers is highly laborious and time consuming with the current wet lab procedures and does not provide full detailed understanding of the binding process. Computational modeling enables one to analyze and understand the binding process and allows one to visualize the natural regression of binding process in a highly controlled environment. Open regions of the aptamers 3D molecular structure provide the binding sites for peptides. Computational modeling can provide a fundamental understanding of the site used and the site changes under varying physiological conditions from the several possible sites.

\section{A. Molecular Dynamics Modeling}

One computational modeling technique applicable for the analysis of bio molecular motion and interactions is based on Molecular Dynamics (MD) modeling [6], [7]. This modeling methodology has been recently applied to determine the chemical, physical and mechanical properties of materials. Molecular Dynamics (MD) modeling is suitable for small systems where individual atoms and or small clusters of atoms are involved and the phenomena influenced by the motion of individual atoms. These models can routinely explore a time scale of picoseconds $\left(10^{-12} s\right)$ to hundreds of nanoseconds. The governing equations in Molecular Dynamics follow classical Newtonian Physics. This method is derived from Newton's equation of motion based on the selected force fields that defines the associated forces in the computational models. This method is popularly used as means to model biological structures and interactions based on the associated molecular configurations. Molecular Dynamics modeling fit the size of most individual biological reactions at the molecular level.

\section{B. Modeling Analysis Methodology}

Molecular Dynamics (MD) modeling analysis to simulate the aptamer - peptide for the MUC1-Anti MUC1 system binding was conducted employing GROMACS, open source $\mathrm{MD}$ analysis software that is one of the fastest molecular dynamics analysis package to date and is commonly used with biomolecules [8]. In this work, we use GROMACS to simulate the dynamics of the aptamer-peptide binding under the same conditions of a $0.15 \mathrm{M}$ solution of sodium chloride $(\mathrm{NaCl})$ that is used in the wet-lab experiments. All simulation analysis was performed with a $0.15 \mathrm{M} \mathrm{NaCl}$ solvent at room temperature $\left(300{ }^{\circ} \mathrm{K}\right)$ and absolute pressure (1 bar). Before binding could be tested, MD simulations using the individual aptamer, and peptide were performed to establish and understand their individual behaviors in this solvent environment. We used the NMR configuration of the Anti-MUC1 aptamer $s 2.2$ for this study. Following the individual analysis, the aptamer-peptide binding was investigated and is focused in the present paper.

The protein structure was minimized for a minimum of $100 \mathrm{ps}$. The potential energy was analyzed to make sure the system reached its most stable configuration. This configuration was then constrained and equilibrated using NVT and NPT ensembles. The NVT equilibration was done with all bonds constrained and temperature coupled by a velocity rescale thermostat which is a modified Berendsen thermostat specific to GROMACS. Pressure constraint in NPT ensemble via the Parrinello-Rahmen barostat allows the simulation cell box to change its shape accordingly. Both NVT and NPT equilibrations were completed for $1 \mathrm{~ns}$ each with the temperature and pressure dynamic variations verified to ensure that the system was indeed equilibrated.

The fully equilibrated system is used as the starting configuration for the MD dynamic analysis. In the dynamic analysis, atoms are unconstrained and are free to move in their most energetically favorable positions in a dynamic process. The molecular system was initially tested for $1 \mathrm{~ns}$ at standard temperature and pressure and was extended to large time duration for the MD dynamic analysis runs for the combined molecular systems consisting of the aptamer and peptide, as the binding process takes a longer time. The aptamer-peptide combinations and aptamer- antibody complex were extended for a total time length of $110 \mathrm{~ns}$ and $15 \mathrm{~ns}$ respectively during the dynamic analysis.

Post processing analysis tools were applied to visualize and quantify the configurations of the system using Visual Molecular Dynamics (VMD) software and GROMACS. We have used several quantities to analyze aptamer-peptide binding. We describe next each of the quantities in detail. Atomic distance was also considered during these post-processing analyses. Spatial confinement and periodic boundary conditions can make visualized data appear unbound as the molecules approach the edges of the box. 
This is to ensure that the distance between the two molecules are remaining constant throughout the simulation further reaffirming that the molecules are still in close proximity. Another parameter that defines the deviation of atomic positions compared to the starting structure is root mean square deviation (RMSD) which is a least squares approximation of the distance between atoms. In the cases of individual atoms, if they are properly settling and are not highly flexible we expect the RMSD to reduce.
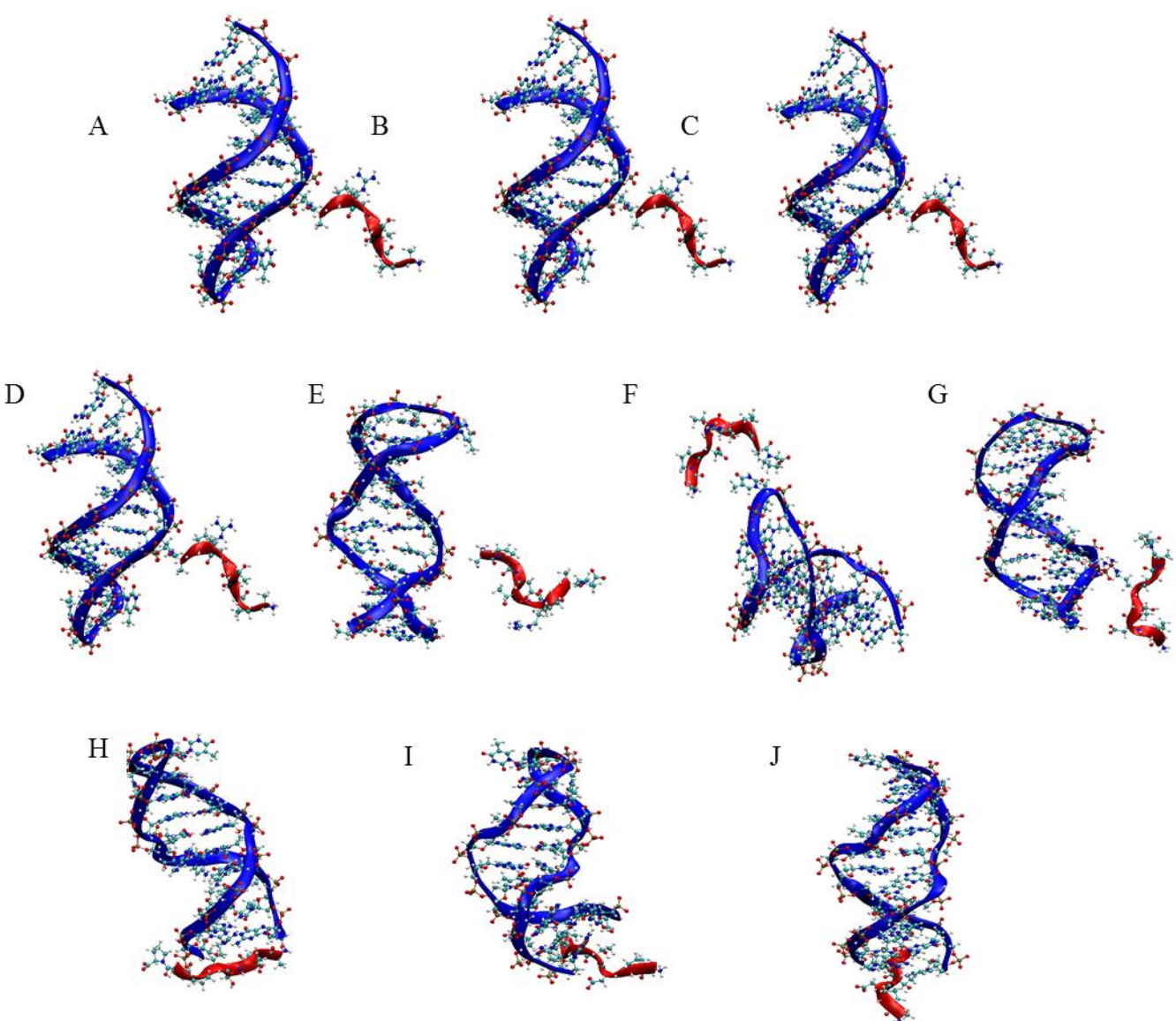

Fig. 1. Anti-MUC1 aptamer (blue) MUC1 peptide (red) visual configurations A) after the addition of ions B) after minimization C) after the NVT Equilibration D) after the NPT equilibration E) at 10ns during simulation F) at $23 n$ s during the simulation $\mathrm{G}$ ) at $35 \mathrm{~ns}$ during the simulation $\mathrm{H}$ ) $45 \mathrm{~ns}$ during the simulation I) at $76 \mathrm{~ns}$ during the simulation $\mathrm{J}$ ) at the end of $110 \mathrm{~ns}$ simulation.

\section{RESULTS AND DISCUSSIONS}

Results and discussions from the MD analysis of aptamerpeptide binding are discussed next.

\section{A. Anti MUC1 Apatamer-MUCl Peptide}

MD analysis of the binding of Anti-MUC1 aptamer and MUC1 peptide is considered first. The visual examination of the molecular configuration revealed little change in the configuration after solvation; minimization and equilibration (see Fig. 1 A- Fig. 1 D). During the dynamic simulation, the aptamer and peptide though initially in close proximity moved apart (see Fig. 1 E). After $25 n s$ the aptamer associated briefly with the $12^{\text {th }}$ Tyrosine residue in the aptamer loop (see Fig. 1 F). The peptide then disassociated before quickly associating with the 5 'end of the aptamer at $44.6 n s$ (see Fig. $1 \mathrm{G}$ ). The peptide is then taken in by the 3' end of the aptamer and is visually parallel to the aptamer (see Fig. $1 \mathrm{H}$ ). The peptide rotates so that it is orthogonal to the aptamer but remains bound to the open 5' and 3' ends of the aptamer throughout the simulation (see Fig. 1 I- Fig. 1 J).

A closer look at the visual configurations during the two associations between the aptamer and peptide reveals that there were selected atoms that interacted more than others (see Fig. 2).
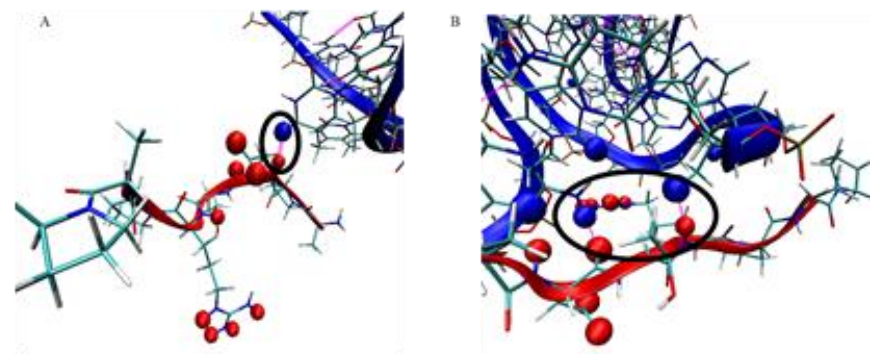

Fig. 2. Anti-MUC1 Aptamer (depicted in blue) and MUC1 peptide (depicted in red) A) during the first association at the 12th Tyrosine residue B) during the second association at the 5 ' and 3 ' ends.

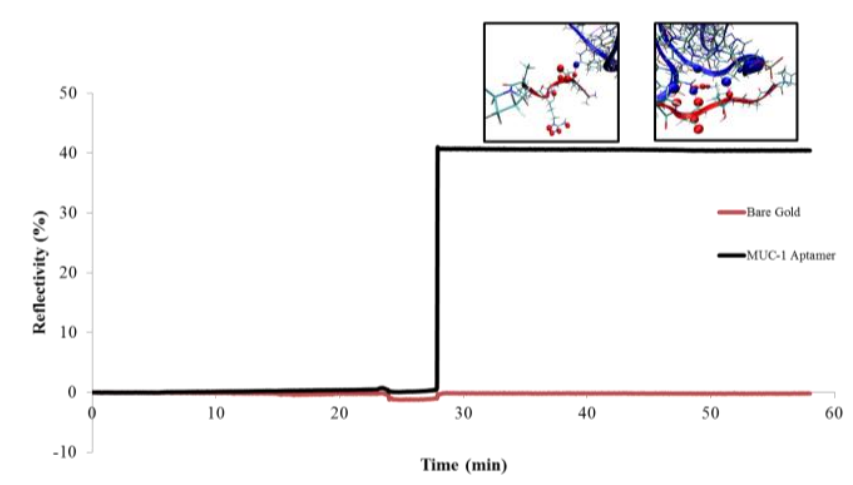

Fig. 3. SPRi analysis of a MUC1 aptamer surface and a flowed sample of MUC1 peptide. 
Fig. 3 shows the Surface Plasmon Resonance Imaging (SPRi) results of an Anti-MUC1 aptamer covered surface interacting with MUC1 peptide. In this, there is a first small peak that indicates the surface effect of surface preparation followed by an increase in the reflectivity as the aptamer and peptide binding. The first association, at the top loop combined with the binding at the 3' and 5' ends of the aptamer observed in the simulations clearly coincide with this increase in reflectivity.

Though the aptamer and peptide appear to be bonded together, a closer examination was needed to confirm the same. A successful binding causes change in energy, RMSD, and bonds. The RMSD showed that the distance between the aptamer and peptide molecules has increased and stabilized after $25 \mathrm{~ns}$. From the visual results 9 peptide and 6 aptamer atoms were identified to be those that continuously participated in binding throughout the MD dynamic analysis simulation. The RMSD of these selected atoms was calculated. The distance between the atoms was found to increase. However theses atoms are interacting at a greater distance than the overall system initially.

The radius of gyration was calculated for the aptamer and peptide and the previously selected 15 atoms. The radius of gyration decreased during the simulation for the aptamer and peptide as well as the selected 15 atoms. The aptamer and peptide became very compact and overall the atoms were 1.4 $\mathrm{nm}$ apart. However the selected atoms were almost $0.5 \mathrm{~nm}$ apart showing how close they were. Since binding occurs with atoms in close proximity, this decrease in the distance indicates that there is binding between the peptide and aptamer.

\section{CONCLUding REMARKS}

Computational modeling and simulations based on molecular dynamics modeling could provide effective means to understand the biomarker aptamer bindings that are present as detection mechanisms in biosensors. Using the GROMACS Molecular Dynamics analysis code, we investigated in the present work Anit-MUC1 aptamer MUC1 peptide binding. The dynamical simulation results were visually and quantitatively analyzed for the conformational changes and the overall behavior of an aptamer and peptide system was observed from a molecular view point, which is not always possible in wet lab experiments. The analysis and visualization of the natural progression of aptamer and peptide binding can aid in biosensor developments that are based on a biomarker aptamer detection mechanism. Preliminary comparison of the dynamic simulation results showed good correlation with the association and disassociation observed in the limited wet lab experiments.

\section{ACKNOWLEDGMENT}

The authors acknowledge the discussions and experimental data from the experimental researchers at Joint School of Nanoscience and Nanoengineering. Research support from Army Research Office under grant W991NF1110168 is acknowledged.

\section{REFERENCES}

[1] B. Strehlitz, N. Nikolaus, and R. Stoltenburg, "Protein detection with aptamer biosensors," Sensors, vol. 8, pp. 4296-4307, 2008.

[2] R. Stoltenburg, C. Reinemann, and B. Strehlitz, "SELEX - a (r) evolutionary method to generate high affinity nucleic acid ligands," Biomolecular Engineering, vol. 24, pp. 381-403, 2007.

[3] A. Baruch, M. Hartmann, M. Yoeli, Y. Adereth, and S. Greenstein, "The breast cancer associated MUC1 gene generates both a receptor and its cognate binding protein," Cancer Research, vol. 59, pp. 1552-1561, 1999.

[4] S. J. Gendler, "The renaissance molecule," Journal of mammary gland biology and neoplasia," vol. 6, pp. 339-353, 2001.

[5] P. Dokurno, P. A. Bate, H. A. Band, L. M. D. Stewart, and J. M. Lally, "Crystal structure at $1.95 \AA$ resolution of the breast tumor specific antibody SM3 complexed with its peptide epitope reveals novel hyper variable loop recognition," Journal of molecular biology, vol. 284, pp. 713-728, 1998.

[6] P. Auffinger and E. Westhof, "Simulations of the molecular dynamics of nucleic acids," Current opinion in structural biology, vol. 8, pp. 227-236, 1998.

[7] P. Jayapal, G. Mayer, A. Heckel, and F. Wenmohs, "Structure-activity relationship of a caged thrombin binding DNA aptamer: Insight gained from molecular dynamics simulation studies," Journal of Structural Biology, vol. 166, pp. 241-250, 2009.

[8] D. Van Der Spoel, E. Lindahl, B. Hess, G. Groenhof, A. E. Mark, and H. J. C. Berendsen, "GROMACS: Fast, flexibe and free," Journal of Computational Chemistry, vol. 26, pp. 1701-1718, 2005.

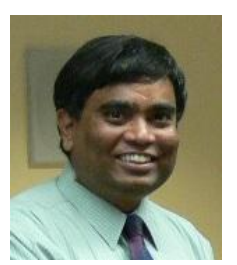

Kristen L. Rhinehardt is a Ph.D. candidate in nanoengineering at Joint School of Nanoscience and Nanogineering at North Carolina A\&T State University. She holds a B.S. degree in Biological Engineering from Cornell University, a MS in Nanoengineering from North Carolina A\&T State University.

Ram V. Mohan is currently an associate professor of nanoengineering at Joint School of Nanoscience and Nanoengineering at North Carolina A\&T State University. He holds a Ph.D. in Mechanical Engineering from University of Minnesota, MS degrees in Theoretical and Applied Mechanics from University of Illinois at Urbana-Champaign and in Mechanical Engineering from West Virginia University. He leads the computational nanoengineering core area.

Goundla Srinivas is a research scientist in the department of nanoengineering at Joint School of Nanoscience and Nanoengineering at North Carolina A\&T State University. He holds a Ph.D. from Indian Institute of Science, Bangalore, India and was a Post-Doctoral researcher at University of Pennsylvania and Center for Molecular Biophysics at Oak Ridge National Laboratory.

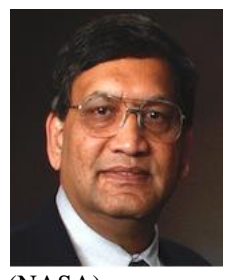

Ajit D. Kelkar is a professor and chairman of nanoengineering at Joint School of Nanoscience and Nanoengineering at North Carolina A\&T State University. He holds Ph.D. in mechanical engineering from Old Dominion University, a MS in mechanical engineering from South Dakota School of Mines. His prior research experience includes working with National Aerospace and Space Administration 